\title{
Errata: Intermediate Valence Behavior of Ternary Cerium and Uranium Transition Metal Borides*
}

\author{
K. N. Yang, M. S. Torikachvili, and M. B. Maple \\ Department of Physics and Institute for Pure and Applied Physical Sciences, \\ University of California at San Diego, La Jolla, California \\ H. C. Ku \\ Department of Physics, National Tsing Hua University, Hsinchu, Taiwan
}

(Received January 3, 1985)

On p. 604 , line $9, \beta$ should be $=12 \pi^{4} N R / 5 \theta_{\mathrm{D}}^{3}(N$ is the number of atoms per formula unit).

On p. 605 , line $1, \theta_{\mathrm{D}}$ should be $=432 \mathrm{~K}$.

On p. 607 , Table I, column $3, \theta_{\mathrm{D}}$ should be as follows:

\begin{tabular}{|c|c|}
\hline Compound & $\theta_{\mathrm{D}}, \mathrm{K}$ \\
\hline $\mathrm{CeCo}_{3} \mathrm{~B}_{2}$ & $505 \pm 11$ \\
\hline $\mathrm{CeRu}_{3} \mathrm{~B}_{2}$ & $251.9 \pm 0.4$ \\
\hline $\mathrm{CeRh}_{3} \mathrm{~B}_{2}$ & $432 \pm 4$ \\
\hline $\mathrm{CeIr}_{3} \mathrm{~B}_{2}$ & $470 \pm 110$ \\
\hline $\mathrm{UCo}_{3} \mathrm{~B}_{2}$ & $345 \pm 7$ \\
\hline $\mathrm{URu}_{3} \mathrm{~B}_{2}$ & $249 \pm 2$ \\
\hline $\mathrm{UIr}_{3} \mathrm{~B}_{2}$ & $282 \pm 4$ \\
\hline
\end{tabular}

On p. 607 , Table I, footnote $b, \theta_{\mathrm{D}}$ should be $\left(12 \pi^{4} N R / 5 \beta\right)^{1 / 3}$.

*This paper appeared in J. Low Temp. Phys. 56, 601 (1984). 